\title{
INFLUENCE OF MAGNETIC FIELD AND WALL SLIP CONDITIONS ON STEADY FLOW BETWEEN PARALLEL FLAT WALL AND A LONG WAYY WALL WTH SORET EFFECT
}

\author{
R. Muthuraj ${ }^{1}$ and S. Srinivas ${ }^{2}$
}

Fluid Dynamics Division, School of advanced Sciences, VIT University, Vellore - 632 014, INDIA.

12email: r.muthu_raj@yahoo.com, ${ }^{2}$ e-mail: sirinusuripeddi@hotmail.com

\begin{abstract}
:
The steady MHD flow in the presence of temperature dependent heat source in a viscous incompressible fluid bounded by a parallel flat wall and a long wavy wall is studied with heat and mass transfer, taking into account the thermal-diffusion (Soret) effects, when the no-slip condition at the channel wall is no longer valid. An external uniform magnetic field and a uniform suction are applied perpendicular to the flat wall. The walls are kept at different but constant temperatures. The velocity, temperature and concentration field have been evaluated numerically for various values of the parameters entering into the problem. The skin friction, rate of heat and mass transfer at the walls are obtained and discussed graphically. It is observed that increasing suction parameter tends to increase concentration of the fluid and thus decreases the fluid velocity. Further, it is interesting to note that when $\gamma=0$ (without slip) and M increases from 0 to 2 there is $28 \%$ decrease in the velocity magnitude, whereas the corresponding decrease, when $\gamma=0.1$ (with slip) is 38\%, which shows that $\gamma$ significantly affect the flow.
\end{abstract}

Keywords: Wavy wall, slip parameter, Sherwood number, suction parameter, Soret number

\section{NOMENCLATURE}

$B_{0} \quad$ transverse magnetic field

$C_{l}^{\prime} \quad$ concentration at the upper wall

$C_{2}^{\prime} \quad$ concentration at the lower wall

d mean width of the channel

$D_{m} \quad$ coefficient of mass diffusivity

$k_{T} \quad$ thermal diffusion ratio

$K \quad$ coefficient of thermal conductivity

$L \quad$ mean free path

$m_{1} \quad$ Maxwell's reflexion coefficient

$M \quad$ Hartmann number

$p \quad$ pressure

$P_{e} \quad$ peclet number

$P_{r} \quad$ Prandtl number

$R \quad$ suction parameter

$S_{r} \quad$ soret number
$T_{2}^{\prime} \quad$ temperature at the lower wall

$T_{1}^{\prime} \quad$ temperature at the upper wall

$T$ temperature distribution

$V_{0} \quad$ suction velocity

\section{Greek symbols}

$\alpha \quad$ heat source parameter

$\gamma \quad$ slip parameter

$\rho \quad$ density

$\sigma \quad$ coefficient of electric conductivity

$\lambda$ non-dimensional frequency parameter

$\nu \quad$ kinematic viscosity

$\varepsilon \quad$ non dimensional amplitude parameter 


\section{Introduction}

When heat and mass transfer occur simultaneously in a moving fluid, the relations between the fluxes and the driving potentials are of more intricate nature. It has been found that an energy flux can be generated not only by temperature gradients but by composition gradients as well. The energy flux caused by a compositions gradient is called the Dufour or diffusion-thermo effect. On the other hand, mass fluxes can also be created by temperature gradients and this is the Soret or thermal-diffusion effect. In general, the thermal-diffusion and diffusion-thermo effects are of a smaller order of magnitude than the effects described by Fourier's or Fick's law and are often neglected in heat and mass transfer processes. However, exceptions are observed therein. Due to the importance of Soret (thermal-diffusion) and Dufour (diffusion-thermo) effects for the fluids with very light molecular weight as well as medium molecular weight many investigators have studied and reported results for these flows [Eckert(1972); Dursunkaya and worek(1992); Streater(2000); Anghel et al.(2000); Postelnicu (2004); Alam et al.(2006); Malashetty (2006); Lakshminarayana et al. (2008); Gaiwad et al. (2009); Srinivas and kothandapani(2008)].

Viscous flow over moving wavy boundaries may be observed in several natural phenomena, viz., the generation of wind waves on water, the formation of sedimentary ripples in river channels and dunes in the desert, etc. The analysis of such flows finds applications in different areas, such as transpiration cooling of reentry vehicles and rocket boosters, cross-hatching on ablative surfaces, and film vaporization in combustion chambers. The subject is also encountered in some industrial applications, e.g., a novel method of fluid transfer, which avoids internal moving parts, employs a duct with flexible walls so as to generate progressive transversal deflection waves. In view of these applications, Lekoudis' et al. (1976) have presented a linear analysis of compressible boundary layer flows over a wavy wall. Shankar and Sinha (1976) studied the Rayleigh problem for a wavy wall. Lessen and Gangawani (1976) studied the effect of small amplitude wall waviness upon the stability of the laminar boundary layer. Bhaskara Reddy and Bathaiah (1981) have discussed the MHD flow of a viscous incompressible fluid between a parallel flat wall and a long wavy wall. In all these problems, the authors have taken the wavy walls to be horizontal. Later, Vajravelu (1989) studied the combined free and forced convection in hydromagnetic flows in a vertical wavy channel with travelling thermal waves. Cho et al. (1998) have studied the problem of linear stability of two-dimensional steady flow in wavy-walled channel. Selvarajan et al. (1998) have numerically reported the analysis of flow in a channel whose walls describe a traveling wave motion. Chamka and Camille (1999) have discussed the problem of mixed convection effects on unsteady flow and heat transfer over a stretched surface. They focused on the effects of mixed convection currents on the problem of unsteady, laminar, boundary-layer flow and heat transfer of an electrically conducting and heat generating or absorbing fluid over a semi-infinite vertical stretched surface in the presence of a uniform magnetic field. Recently, Srinivas and Muthuraj $\left(2010^{\mathrm{a}}\right)$ have discussed the effects of thermal radiation and space porosity on MHD mixed convection flow in a vertical channel using homotopy analysis method. More recently, Muthuraj and Srinivas $\left(2010^{b}\right)$ have studied MHD oscillatory flow of an optically thin fluid in an asymmetric wavy channel filled with porous medium.

In several applications, the flow pattern corresponds to a slip flow, the fluid presents a loss of adhesion at the wetted wall making the fluid slide along the wall. When the molecular mean free path length of the fluid is comparable to the distance between the plates as in nanochannels or microchannels, the fluid exhibits noncontinuum effects such as slip-flow as demonstrated experimentally by Derek et al.(2002). Nearly, 200 years ago Navier (1823) proposed a general boundary condition that permits the possibility of fluid slip at a solid boundary. This boundary condition assumes that the tangential velocity of the fluid relative to the solid at a point on its surface is proportional to the tangential stress acting at that point. Barrat and Bocquet (1999) have used molecular dynamics to compute slip for liquids and Pit et al. (2000) have measured slip for hexadecane on several modified sapphire surface using a rotating disk. Neill et al. (1986) used a linear slip, Basset-type (1961), boundary condition to remove the contact-line singularity that would otherwise prevent the movement of a halfsubmerged sphere normal to planner free surface bounding a semi-infinite viscous fluid. Hron et al. (2008) have presented analytical solutions for the flows of a generalized fluid of complexity two in special geometries under the assumption that the flows meet Navier slip conditions at the boundary. Ali et al. (2008) have studied the slip effects on the peristaltic transport of MHD fluid with variable viscosity. Ebaid (2008) studied the effects of magnetic field and wall slip conditions on the peristaltic transport of a Newtonian fluid in an asymmetric channel. Sirnivas and Muthuraj $\left(2010^{\mathrm{b}}\right)$ have examined MHD flow with slip effects and temperature dependent heat source in a vertical wavy porous space. More recently, Muthuraj and Srinivas $\left(2010^{\mathrm{a}}\right)$ have investigated the problem of mixed convection heat and mass transfer flow through a vertical wavy porous space with traveling thermal waves. However, no work has been reported yet regarding the influence of heat and mass transfer on 
the MHD flow through a horizontal wavy walled channel with slip effects. With the above discussion in mind, we put forward the MHD flow of a viscous fluid between a parallel flat wall and a long wavy wall in the presence of a slip condition taking into account the thermal-diffusion (Soret) effects. The fluid is sucked through the wall $\mathrm{y}=0$ with the constant suction velocity $V_{0}$. The effects of pertinent parameters entering into the problem have been discussed in detail. The organization of the paper is as follows: Problem is formulated in Section 2. Section 3 deals with the solution of the problem. Numerical results and discussion are given in Section 4. The conclusions have been summarized in Section 5.

\section{Formulation of the Problem}

Consider the steady, incompressible and MHD flow of a viscous fluid through a non-isothermal parallel flat wall and a long wavy wall (see Fig.1). The $\mathrm{x}$ - axis is taken along the parallel flat wall and a straight line perpendicular to that as the y-axis, so that the wavy wall is represented by $y=d+\varepsilon^{*} \cos k x$ and the flat wall by $y=0$. A uniform magnetic field is applied in the direction normal to the walls. The wavy and flat walls are maintained at constant temperatures of $T_{1}^{\prime}$ and $T_{2}^{\prime}$ respectively.

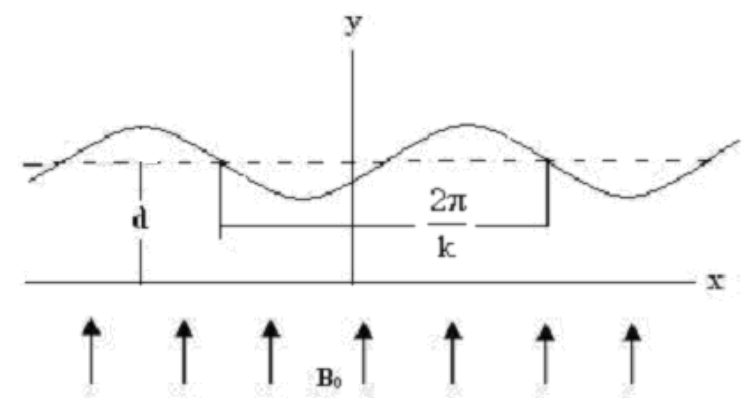

Fig. 1: Flow geometry of the problem

The governing equations for this problem are based on the balance laws of mass, linear momentum, energy and concentration modified to account for the presence of the magnetic field and temperature dependent heat source effects. These can be written as

$$
\begin{aligned}
& \frac{\partial u}{\partial x}+\frac{\partial v}{\partial y}=0 \\
& \rho\left(u \frac{\partial u}{\partial x}+v \frac{\partial u}{\partial y}\right)=-\frac{\partial p}{\partial x}+\mu\left(\frac{\partial^{2} u}{\partial x^{2}}+\frac{\partial^{2} u}{\partial y^{2}}\right)-\sigma B_{0}^{2} \mu_{e} u \\
& \rho\left(u \frac{\partial v}{\partial x}+v \frac{\partial v}{\partial y}\right)=-\frac{\partial p}{\partial y}+\mu\left(\frac{\partial^{2} v}{\partial x^{2}}+\frac{\partial^{2} v}{\partial y^{2}}\right) \\
& \rho C_{p}\left(u \frac{\partial T}{\partial x}+v \frac{\partial T}{\partial y}\right)=K\left(\frac{\partial^{2} T}{\partial x^{2}}+\frac{\partial^{2} T}{\partial y^{2}}\right)+Q\left(T-T_{1}^{\prime}\right) \\
& \left(u \frac{\partial C}{\partial x}+v \frac{\partial C}{\partial y}\right)=D_{m}\left(\frac{\partial^{2} C}{\partial x^{2}}+\frac{\partial^{2} C}{\partial y^{2}}\right)+\frac{D_{m} k_{T}}{T}\left(\frac{\partial^{2} T}{\partial x^{2}}+\frac{\partial^{2} T}{\partial y^{2}}\right)
\end{aligned}
$$

The boundary conditions of the problem are

$$
\begin{aligned}
& u=L_{1}\left(\frac{\partial u}{\partial y}\right) \quad v=-V_{0} \quad T=T_{1}^{\prime} \quad C=C_{1}^{\prime}, \quad \text { at } \quad y=0 \\
& u=-L_{1}\left(\frac{\partial u}{\partial y}\right) \quad v=0 \quad T=T_{2}^{\prime} \quad C=C_{2}^{\prime}, \quad \text { at } \quad y=d+\varepsilon^{*} \cos k x
\end{aligned}
$$


Where, $L_{1}=\left[\frac{2-m_{1}}{m_{l}}\right] L, L$ is the mean free path, $m_{l}$ is the Maxwell's reflexion coefficient, $B_{0}$ is the transverse magnetic field, $D_{m}$ is the coefficient of mass diffusivity, $d$ is mean width of the channel, $p$ is the pressure, $T$ is the temperature distribution, $\rho$ is the density, $v$ is the kinematic viscosity, $K$ is the coefficient of thermal conductivity, $\sigma$ is the coefficient of electric conductivity, $k_{T}$ is the thermal diffusion ratio, $T_{1}^{\prime}$ and $T_{2}^{\prime}$ are the wall temperatures, $C_{1}^{\prime}$ and $C_{2}^{\prime}$ are the wall concentrations, $\bar{T}$ is the mean value of $T_{1}^{\prime}$ and $T_{2}^{\prime}$.

Since the flat wall is infinite in length, $\frac{\partial u}{\partial x}=0$

Integrating eqn(1) and using eqn(6), we obtain $v=-V_{0}$

We introduce the non-dimensional variables

$$
\left(x^{*}, y^{*}\right)=\frac{1}{d}(x, y),\left(u^{*}, v^{*}\right)=\frac{1}{V_{0}}(u, v), p^{*}=\frac{p d}{\mu_{0} V_{0}}, T^{*}=\frac{T-T_{1}^{\prime}}{T_{2}^{\prime}-T_{1}^{\prime}}, \phi^{*}=\frac{C-C_{1}^{\prime}}{C_{2}^{\prime}-C_{1}^{\prime}}
$$

In view of eqns (9) and (10), eqns (2)-(5) reduce to (omitting * symbols for clarity)

$$
\begin{aligned}
& \frac{d^{2} u}{d y^{2}}+R \frac{d u}{d y}-M^{2} u=\frac{d p}{d x} \\
& \frac{d p}{d y}=0 \\
& \frac{d^{2} T}{d y^{2}}+P_{r} R \frac{d T}{d y}+\alpha T=0 \\
& \frac{d^{2} \phi}{d y^{2}}+P_{e} \frac{d \phi}{d y}+S_{r} \frac{d^{2} T}{d y^{2}}=0
\end{aligned}
$$

Together with boundary conditions,

$$
\begin{aligned}
& u=\gamma u^{\prime}, \quad v=-1, \quad T=0, \quad \phi=0 \quad \text { at } \quad y=0 \\
& u=-\gamma u^{\prime}, \quad v=0, \quad T=1, \quad \phi=1 \quad \text { at } \quad y=h
\end{aligned}
$$

Where $h=1+\varepsilon \cos \lambda x, M^{2}=\sigma B_{0}^{2} \mu_{e}^{2} d^{2} / \mu$ is the Hartmann number, $P_{r}=\mu C_{p} / K$ is the Prandtl number, $v=\mu / \rho$ is the kinematic viscosity, $\varepsilon=\varepsilon^{*} / d$ is the non-dimensional amplitude parameter $(\varepsilon<<1), \lambda(=k d)$ is the nondimension frequency parameter, $R=V_{0} d / v$ is the suction parameter, $\alpha=Q d^{2} / K$ is the heat source parameter, $P_{e}=V_{0} d / D_{m}$ is the Peclet number and $S_{r}=k_{T}\left(T_{2}^{\prime}-T_{1}^{\prime}\right) / \bar{T}\left(C_{2}^{\prime}-C_{1}^{\prime}\right)$ is the Soret number, $\gamma=L_{1} / d$ is the slip parameter. From eqn.(12), we observe that the fluid pressure $p$ is independent of $y$. We assume that the pressure gradient $\frac{d p}{d x}$ is constant.

\section{Method of solution}

Solving eqn. (11) using the boundary conditions (15)-(16), we obtain

$u(y)=\frac{c}{M^{2}}\left(\frac{\left[\left(e^{\beta_{4} h}-1\right)+\gamma \beta_{4}\left(1+e^{\beta_{4} h}\right)\right] e^{\beta_{3} y}-\left[\left(e^{\beta_{3} h}-1\right)+\gamma \beta_{3}\left(1+e^{\beta_{3} h}\right)\right] e^{\beta_{4} y}}{\left(1-\gamma^{2} \beta_{3} \beta_{4}\right)\left(e^{\beta_{4} h}-e^{\beta_{3} h}\right)+\gamma\left(e^{\beta_{3} h}+e^{\beta_{4} h}\right)\left(\beta_{4}-\beta_{3}\right)}-1\right)$

Making use of eqns. (15)-(16) solve the eqn. (13), we obtain

$T(y)=\frac{e^{\beta_{1} y}-e^{\beta_{2} y}}{e^{\beta_{1} h}-e^{\beta_{2} h}}$

Incorporating eqn. (18) into (14) then solve, we get

$\phi(y)=\frac{\left(1-e^{-P_{e} y}\right)}{\left(1-e^{-P_{e} h}\right)}+\frac{S_{r}}{e^{\beta_{1} h}-e^{\beta_{2} h}}\left(\frac{\beta_{1}\left(1-e^{\beta_{1} y}\right)}{P_{e}+\beta_{1}}-\frac{\beta_{2}\left(1-e^{\beta_{2} y}\right)}{P_{e}+\beta_{2}}\right)$ 


$$
+\frac{S_{r}\left(1-e^{-P_{e} y}\right)}{\left(e^{\beta_{1} h}-e^{\beta_{2} h}\right)\left(1-e^{-P_{e} h}\right)}\left(\frac{\beta_{2}\left(1-e^{\beta_{2} y}\right)}{P_{e}+\beta_{2}}-\frac{\beta_{1}\left(1-e^{\beta_{1}^{y}}\right)}{P_{e}+\beta_{1}}\right)
$$

Where, $c=\frac{d p}{d x} ; \beta_{1}=\frac{-P_{r} R+\sqrt{P_{r}^{2} R^{2}-4 \alpha}}{2} ; \beta_{2}=\frac{-P_{r} R-\sqrt{P_{r}^{2} R^{2}-4 \alpha}}{2} ; \beta_{3}=\frac{-R+\sqrt{R^{2}+4 M^{2}}}{2}$; $\beta_{4}=\frac{-R-\sqrt{R^{2}+4 M^{2}}}{2}$

The shear stress at any point in the fluid is given by $\bar{\tau}_{x y}=\mu\left(\frac{\partial u}{\partial y}+\frac{\partial v}{\partial x}\right)$

In non dimensionless form $\tau=\left(\frac{d}{\mu V_{0}}\right) \bar{\tau}_{x y}=\frac{\partial u}{\partial y}$

The skin friction at the flat wall $y=0$ and the wavy wall $y=h$ is given by

$$
\tau_{0}=\left(\frac{\partial u}{\partial y}\right)_{y=0} ; \tau_{l}=\left(\frac{\partial u}{\partial y}\right)_{y=h}
$$

The heat transfer coefficient, characterized by Nusselt number $(N u)$ on the tube boundary is

$$
N u=-K \frac{\partial T}{\partial y}
$$

In dimensionless form it becomes

$$
N u=-K\left(\frac{T_{2}^{\prime}-T_{1}^{\prime}}{d}\right)\left(\frac{\partial T}{\partial y}\right)
$$

The Nusselt number at the flat wall $y=0$ and the wavy wall $y=h$ is given by

$$
N u_{0}=(N u)_{y=0} ; N u_{1}=(N u)_{y=h}
$$

The dimensionless mass transfer number corresponding to the Nusselt number is the Sherwood number, written

as, $S h=\frac{\partial \phi}{\partial y}$

The Sherwood number at the flat wall $y=0$ and the wavy wall $y=h$ is given by

$$
S h_{0}=\left(\frac{\partial \phi}{\partial y}\right)_{y=0} ; S h_{l}=\left(\frac{\partial \phi}{\partial y}\right)_{y=h}
$$

\section{Results and Discussion}

In order to get a physical insight into the problem, factors such as velocity, temperature, concentration, skin friction and Nusselt number have been discussed by assigning numerical values to various parameters obtained in the mathematical formulation of the problem and the results are graphically shown in Figs.2-7. The graphical analysis shows that the slip parameter $(\gamma)$, Soret number $\left(S_{r}\right)$, Peclet number $\left(P_{e}\right)$, Prandtl number $\left(P_{r}\right)$, suction parameter $(R)$, Hartmann number $(M)$ and heat source parameter $(\alpha)$ play an important role in this discussion about characteristics of the dynamical flow patterns. Throughout the computations we employ $P_{r}=0.5, S_{r}=0.5, P_{e}=1, \alpha=5, R=0.5, \mathrm{c}=1, \varepsilon=0.02, x=1$ and $M=2$, unless otherwise stated. The velocity distribution is graphed in Fig.2 for different values of the parameters $\gamma, R$ and $M$. Fig.2a shows that increasing slip parameter lead to increase the fluid velocity. The effect of suction parameter on velocity distribution is graphed in Fig.2 (b). It is seen from this figure that the velocity profiles decrease monotonically with the increase of suction parameter indicating the usual fact that suction stabilizes the boundary layer growth. From Fig.2c, one can notice that increasing Hartmann number tends to decrease the fluid velocity. It is because that the application of transverse magnetic field will result a resistive type force (Lorentz force) similar to drag force which tends to resist the fluid flow and thus reducing its velocity. Further, it is interesting to note that when $\gamma=0$ (without slip) and $\mathrm{M}$ increases from 0 to 2 there is $28 \%$ decrease in the velocity value, whereas the 
corresponding decrease, when $\gamma=0.1$ (with slip) is $38 \%$, which shows that $\gamma$ significantly affect the flow. The solution of temperature distribution (T) is shown in Fig. 3 for different values of $\alpha$ and $R$ with fixed values of all other parameters.
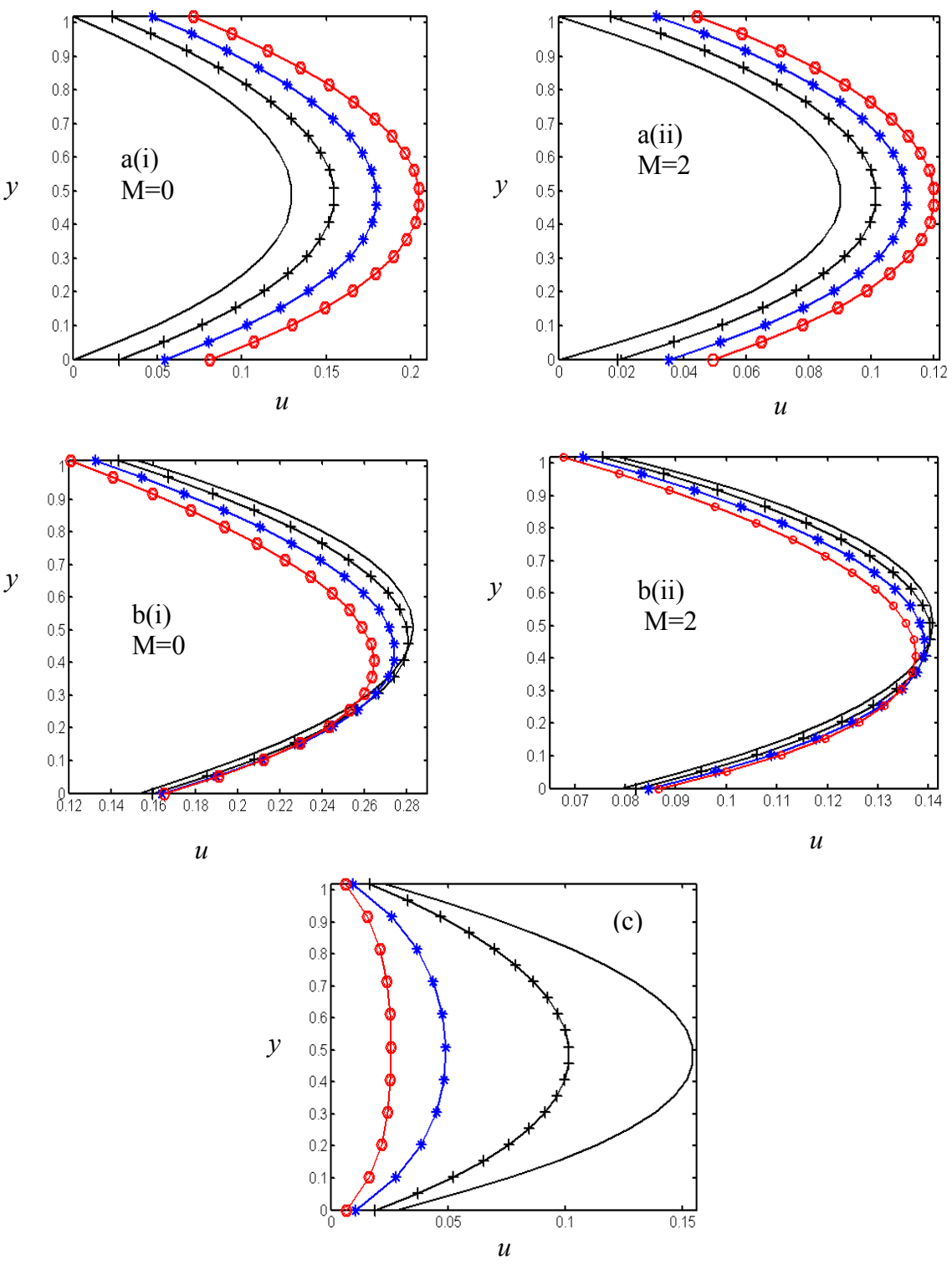

Fig.2 Velocity distribution

$$
\begin{gathered}
\mathrm{a}:{ }_{-} \gamma=0,{ }_{-} \gamma=0.05,{ }_{-}^{*} \gamma=0.1, o_{-} \gamma=0.15 \\
\mathrm{~b}: R_{-}=0,{ }_{+} R=0.5,{ }^{*}{ }_{-} R=1, o_{-} R=1.5, \gamma=0.3 \\
\mathrm{c}:{ }_{-} M=0,{ }_{-} M=2,{ }_{-} M=4, o_{-} M=6, \gamma=0.05,
\end{gathered}
$$

Fig.3a is graphed to see the influence of $\alpha$ on temperature distribution. It is well known that the heat generation causes the fluid temperature to increase, which has tendency to increase the thermal buoyancy effects. From Fig.3b, we observe that increasing suction parameter lead to enhance fluid temperature. Further, it is seen that increasing $\mathrm{R}$ temperature increases significantly in the presence of heat generation (i.e. $\alpha>0$ ). 
On the other hand heat absorption (i.e. $\alpha<0$ ) produces opposite effect. Fig.4 depicts the behavior of the concentration distribution $(\phi)$ for different values of $S_{r}, R, P_{e}$ and $P_{r}$. From this figure, we see that the fluid concentration decreases with increasing $S_{r}, P_{e}$ and $P_{r}$ whereas it enhance by increasing suction parameter.
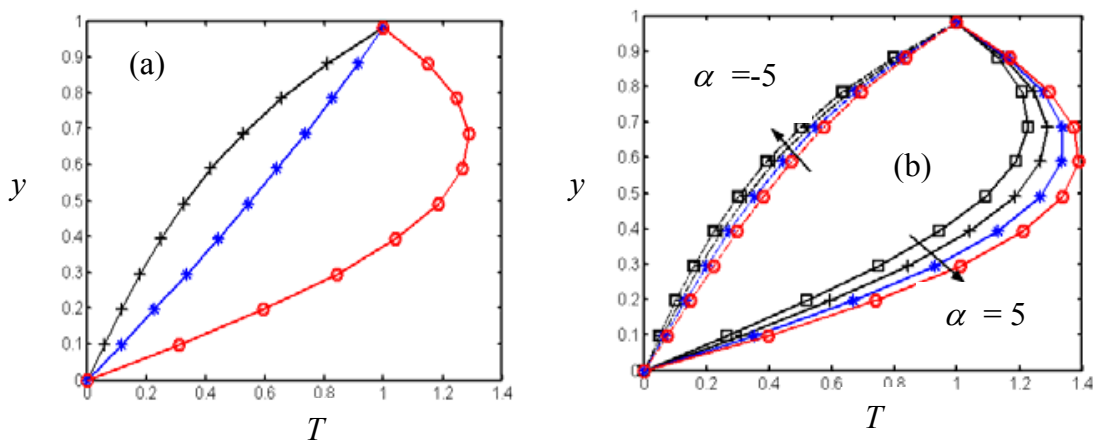

Fig.3 Temperature distribution

$\mathrm{a}:{ }_{+} \alpha=-5,{ }^{*} \alpha=0$, o_ $_{-} \alpha=5$

b: $\square R=0,+R=0.5, * R=1$, o $R=1.5$
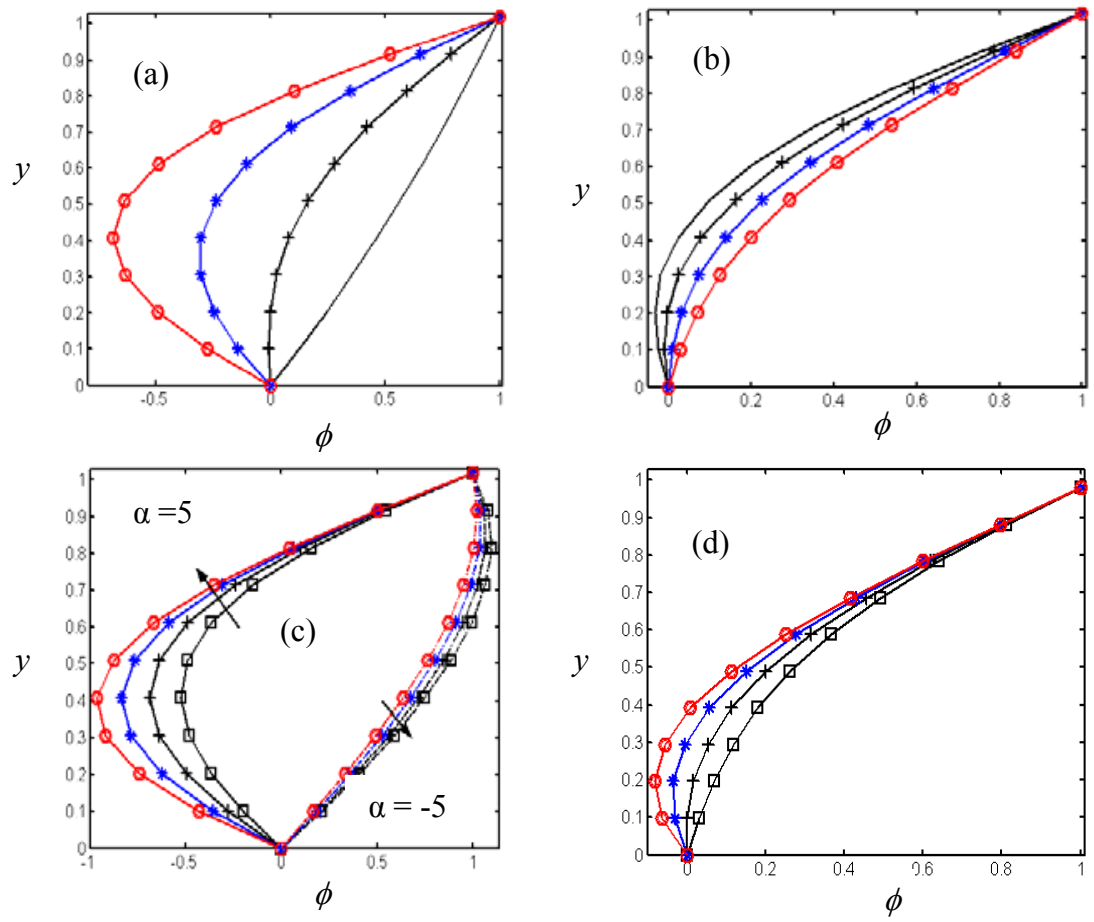

Fig.4 Concentration distribution

$$
\begin{aligned}
& \mathrm{a}: \_S_{r}=0,{ }_{-} S_{r}=0.5,{ }^{*}{ }_{-} S_{r}=1, \mathrm{o}_{-} S_{r}=1.5 \mathrm{~b}:{ }_{-} R=0,{ }_{+}+R=0.5,{ }^{*}{ }_{-} R=1, \mathrm{o}_{-} R=1.5 \\
& \mathrm{c}: P_{e}=0,{ }_{-} P_{e}=0.5,{ }^{*} P_{e}=1, o_{-} P_{e}=1.5 \quad \mathrm{~d}: \square_{-} P_{r}=0,{ }_{-} P_{r}=0.5,{ }^{*} P_{r}=1, o_{-} P_{r}=1.5
\end{aligned}
$$

Also, we note that concentration of the fluid is positive and decreasing with an increase of $P_{e}$ when $\alpha<0$ (heat absorption) whereas it is negative when $\alpha>0$ (heat generation). The effect of $P_{r}$ and $R$ on Nusselt number distribution is plotted in Fig.5. It depicts that $N u$ increases with an increase of $\alpha$ and $P_{r}$ at the flat wall $y=0$, but this behavior is reversed at the wavy wall $y=h$. Physically, it means that the heat can sometimes 
flow out of and other times into either wall. These behaviors are all valid qualitatively for air $\left(P_{r}=0.71\right)$ and water $\left(P_{r}=7\right)$. Similar result can be noticed in Fig.5b if $P_{r}$ is replaced by $R$.
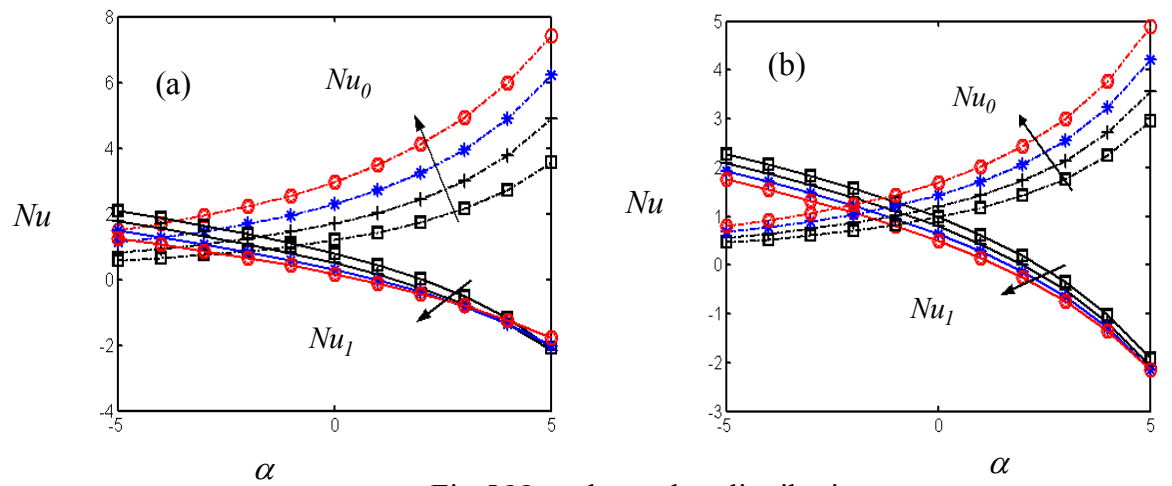

Fig. 5 Nusselt number distribution

a: $\square_{-} P_{r}=0,{ }_{-} P_{r}=1,{ }^{*} P_{r}=3, o_{-} P_{r}=5, R=0.5, M=2, \gamma=0.5, S_{r}=0.5, P_{e}=1$

b: $\square_{-} R=0,{ }_{-} R=0.4,{ }^{*}{ }_{-} R=0.8$, o_$_{-} R=1.2, M=2, \gamma=0.5, S_{r}=0.5, P_{r}=0.5, P_{e}=1$

$\tau$

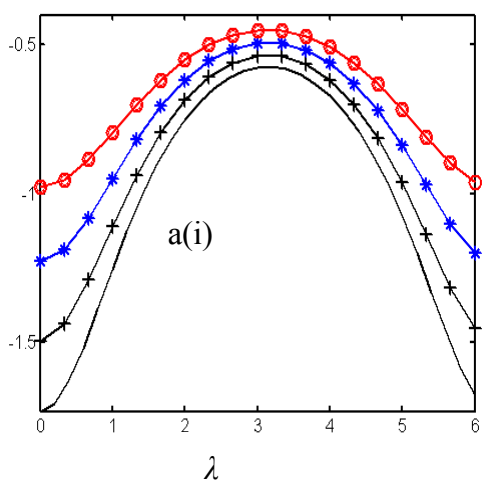

$\tau$

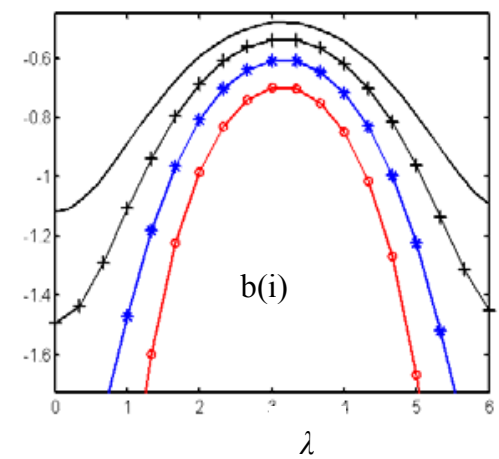

$\tau$
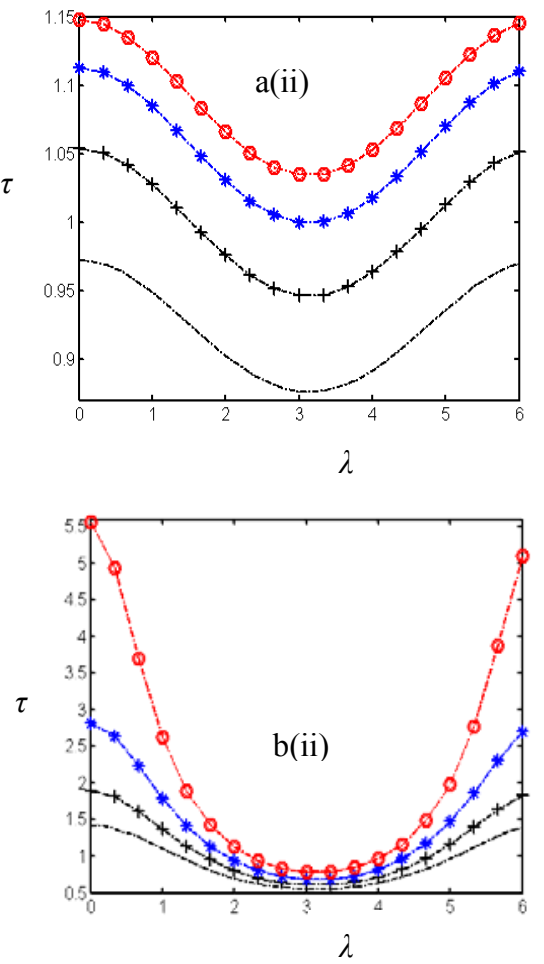

Fig.6 Skin friction distribution (_ at the wavy wall, _... at the flat wall )

a: $\_R=0,{ }_{-} R=0.5,{ }^{*}{ }_{-} R=1, o_{-} R=1.5, M=2, \gamma=0.05, S_{r}=0.5, P_{r}=0.5, P_{e}=1$

b: ${ }_{-} \gamma=0,{ }_{-} \gamma=0.05,{ }^{*} \gamma=0.1, o_{-} \gamma=0.15, M=2, \mathrm{R}=0.5, S_{r}=0.5, P_{r}=0.5, P_{e}=1$

Fig.6 is plotted to see the effects of $R$ and $\gamma$ on the skin friction at the walls. In Fig.6a, we see that magnitude of skin friction increases with an increase of $R$ at both the walls. The influence of $\gamma$ on skin friction at both the walls appears in Fig. 6b. It is observed that skin friction decreases at the wall $y=h$ while it increases at the other wall with an increase of $\gamma$. It means that the flow retards at both the walls. 


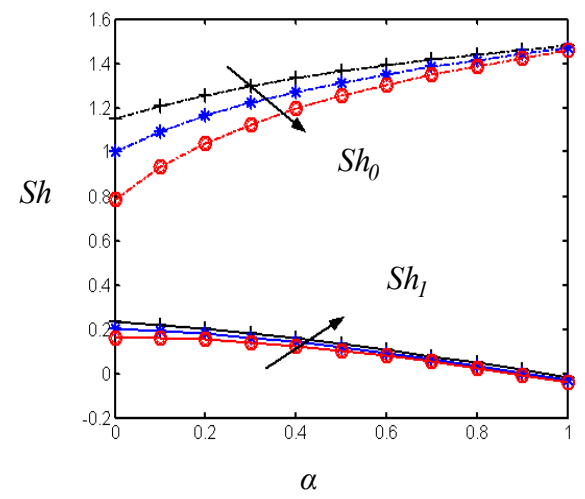

Fig.7 Sherwood number distribution

$$
{ }_{-} R=0.2,{ }_{-}{ }_{-} R=0.4, \mathrm{o}_{-} R=0.6, M=2, \gamma=0.5, \mathrm{~S}_{\mathrm{r}}=0.5, P_{r}=0.5, P_{e}=1
$$

Fig.7 shows the variation in Sherwood number distribution $(S h)$ with $\alpha$ for different values of $R$. From this figure, we notice that Sherwood number decreases with an increase of $R$ while it increases with increasing $\alpha$ at the flat wall $y=0$. Further, we observe that Sherwood number decreases with an increase of $\alpha$ and $R$ at the other wall.

\section{Conclusion}

The influence of applied magnetic field and wall slip effect on the MHD flow between a parallel flat wall and a long wavy wall has been analyzed. The analytical expressions are constructed for velocity, temperature and concentration. The effect of pertinent parameters on flow, heat and mass transfer characteristics are discussed in detail. The salient observations of the present study are listed below.

i) The effect of increasing suction parameter suppresses the velocity while it enhances the fluid temperature.

ii) Increasing $S_{r}, P_{e}$ and $P_{r}$ tends to reduce the fluid concentration whereas $R$ lead to increase the concentration of the fluid.

iii) Nusselt number increases with increasing values of $P_{r}$ and $R$ at the flat wall $y=0$ but this behavior is reversed at the wavy wall $y=h$.

iv) The skin friction $(\tau)$ enhances at both the walls with increasing $R$. Further, we observe that skin friction decreases with increasing slip parameter at the wavy wall $y=h$ while the opposite is true at the other wall.

\section{References}

Alam, M. S., Rahman, M. M. and Samad, M. A. (2006): Numerical study of the combined free forced convection and mass transfer flow past a vertical porous plate in a porous medium with heat generation and thermal diffusion, Nonlinear anal model control, Vol. 11, pp. 331-343.

Ali, N., Hussain, Q., Hayat, T. and Asghar, S. (2008): Slip effects on the peristaltic transport of fluid with variable viscosity. Phy Letters A, Vol. 372,pp. 1477-1489. doi:10.1016/j.physleta.2007.09.061

Anghel. M., Takhar. H.S. and Pop, I. (2000): Dufour and Soret effects on free-convection boundary layer over a vertical surface embedded in a porous medium, Studia Universitaties Babes- Bolyai, Mathematica, Vol.XLV, pp. 11-21.

Barrat, J. and Bocquet, L. (1999): Large slip effect at a non wetting fluid-solid interface. Phys Rev Lett, Vol.82, pp. 4671-4674. doi:10.1103/PhysRevLett.82.4671

Basset, A. B. (1961): A Treatise on Hydro dynamics, Vol. 2, Dover New York. Bhaskara Reddy, N. and Bathaiah, D. (1981): Magneto Hydrodynamic flow of a viscous incompressible fluid between a Parallel Flat Wall and a Long wavy wall. Def. Sci J, Vol. 31, pp. 315-322. Chamkha, A. J. and Camille, I. (1999): Mixed convection effects on unsteady flow and heat transfer over a stretched surface, Int Comm Heat Mass Transfer, Vol. 26, pp. 717- 727. doi:10.1016/S0735-1933(99)00058-5 
Cho, K.J., Kim, M. U. and Shin, H. D. (1998): Linear stability of two -dimensional steady flow in wavy walled channels, Fluid Dynamics Research Vol. 23, pp.349- 370. doi:10.1016/S0169-5983(98)00003-3

Derek, C., Tretheway, D. C. and Meinhart, C. D. (2002): Apparent fluid slip at hydrophibic micro channel walls, Phys Fluids, Vol. 14, L9-L12. doi:10.1063/1.1432696

Dursunkaya, Z. and Worek, W. M. (1992): Diffusion-thermo and thermal-diffusion effects in transient and steady natural convection from vertical surface, Int J Heat Mass Transfer, Vol. 35, pp. 2060-2065. doi:10.1016/0017-9310(92)90208-A

Ebaid, A. (2008): Effects of magnetic field and wall slip conditions on the peristaltic transport of a Newtonian fluid in an asymmetric channel, Phys. Letters, A Vol. 372, pp. 4493-4499. doi:10.1016/j.physleta.2008.04.031

Eckert, E. R. G. and Drake, R. M. (1972): Analysis of Heat and Mass Transfer, McGraw- Hill, NewYork. Gaikwad, S. N., Malashetty, M. S. and Rama Prasad, K. (2009): An analytical study of linear and non-linear double diffusive convection in a fluid saturated anisotropic porous layer with Soret effect, Appl Math Modelling, Vol.33, pp. 3617-3635. doi:10.1016/j.apm.2008.12.013

Hron, J., Roux, C. L., Malik, J. and Rajagopal, K. R. (2008): Flows of incompressible fluids subject to Navier's slip on the boundary, Comput. Math. Appl, Vol. 56, pp. 2128-2143. doi:10.1016/j.camwa.2008.03.058

Lakshmi Narayana, P. A., Murthy, P. V. S. N. and Gorla, R. S. R. (2008): driven thermo solutal convection induced by inclined thermal and solutal gradients in a shallow horizontal layer of a porous medium, J Fluid Mech., Vol. 612, pp. 1-19.

Lekoudis, S. G., Nayfeh, A.H. and Saric, W.S. (1976): Compressible Boundary Layers over Wavy Walls, Phys Fluids, Vol. 19, pp. 514-519. doi:10.1063/1.861507

Lessen, M. and Gangwani, S. T(1976): Effect of small amplitude wall waviness upon the stability of the laminar boundary layer. Phys Fluids, Vol. 19, pp. 510-513. doi:10.1063/1.861515

Malashetty M. S., Gaikwad S. N. and Swamy M. (2006): An analytical study of linear and non-inear double diffusive convection with Soret effect in couple stress liquids. Int J Thermal Sci, Vol. 45, pp. 897-907. doi:10.1016/j.ijthermalsci.2005.12.005

Muthuraj, R. and Srinivas. S. (2010a): Mixed convective heat and mass transfer in a vertical wavy channel with traveling thermal waves and porous medium, Computers and Mathematics with Applications, Vol. 59, pp. 35163528. doi:10.1016/j.camwa.2010.03.045

Muthuraj, R. and Srinivas. S. (2010b): A Note on Heat transfer to MHD oscillatory flow in an asymmetric wavy channel, Int commun Heat Mass transfer (Accepted).

Navier, C. L. M. H. (1823): Memoire sur les lois du mouvement des fluids, Mem Acad R Sc. Inst France, Vol. 1, pp. 414-421.

O’Neill, M. E., Ranger, K. B. and Brenner, H. (1986): Slip at the surface of a translating- rotating sphere. Phys Fluids, Vol. 29, pp. 913-924.

Pit, R., Hervet, H. and Leger, L.(2000): Direct experimental evidence of slip in hexadecane: Solid interfaces, Phy Rev Lett, Vol.85, pp. 980-983. doi:10.1103/PhysRevLett.85.980 PMid:10991454 Postelnicu, A. (2004): Influence of a magnetic field on heat and mass transfer by natural convection from vertical surfaces in porous media considering Soret and Dufour effects, Int J Heat Mass Transfer, Vol. 47, pp.1467-1472. doi:10.1016/i.ijheatmasstransfer.2003.09.017

Selvarajan, S., Tulapurkara, E.G. and Vasanta Ram, V. (1998): A numerical simulation of flow through wavy walled channels. Int. J. Num. Fluids, Vol. 26, pp. 519-531. doi:10.1002/(SICI)10970363(19980315)26:5<519::AID-FLD630>3.0.CO;2-C

Shankar, P. N. and Sinha, U. N. (1976): The Rayleigh problem for a wavy wall, J Fluid mech, Vol. 77, pp. 243256. doi:10.1017/S0022112076002097

Srinivas, S. and Kothandapani, M. (2009): The influence of heat and mass transfer on MHD peristaltic flow through a porous space with compliant walls, Appl. Math Compt., Vol. 213, pp. 197-208. doi:10.1016/j.amc.2009.02.054

Srinivas. S, Muthuraj, R. (2010a): Effects of thermal radiation and space porosity on MHD mixed convection flow in a vertical channel using homotopy analysis method, Commun Nonlinear Sci Numer Simulat., Vol.15, pp. 2098-2108. doi:10.1016/j.cnsns.2009.09.003

Srinivas, S. and Muthuraj, R. (2010b), MHD flow with slip effects and temperature dependent heat source in a vertical wavy porous space, Chemical Engineering communication, Vol. 197, pp.1387-1403. doi:10.1080/00986441003626102

Streater, R. F. (2000): The Soret and Dufour effects in statistical dynamics, Proc R Soc. Lond A, Vol. 456, pp. 205-221. doi:10.1098/rspa.2000.0514

Vajravelu, K. (1989): Combined free and forced convection in hydromagnetic flows in Vertical wavy channel with traveling thermal waves, Int J Engg. Sci, Vol. 278, pp. 289-300. 